\title{
lodinated contrast medium: Is there a re(n)al problem? A clinical vignette-based review
}

\author{
Karim Lakhal ${ }^{1 *} \mathbb{D}$, Stephan Ehrmann ${ }^{2}$ and Vincent Robert-Edan ${ }^{1}$
}

\begin{abstract}
As we were taught, for decades, that iodinated contrast-induced acute kidney injury should be dreaded, considerable efforts were made to find out effective measures in mitigating the renal risk of iodinated contrast media. Imaging procedures were frequently either downgraded (unenhanced imaging) or deferred as clinicians felt that the renal risk pertaining to contrast administration outweighed the benefits of an enhanced imaging. However, could we have missed the point? Among the abundant literature about iodinated contrast-associated acute kidney injury, recent meaningful advances may help sort out facts from false beliefs. Hence, there is increasing evidence that the nephrotoxicity directly attributable to modern iodinated CM has been exaggerated. Failure to demonstrate a clear benefit from most of the tested prophylactic measures might be an indirect consequence. However, the toxic potential of iodinated contrast media is well established experimentally and should not be overlooked completely when making clinical decisions. We herein review these advances in disease and pathophysiologic understanding and the associated clinical crossroads through a typical case vignette in the critical care setting.

Keywords: Contrast media (MeSH: D003287), Intensive care units (MeSH D007362), Drug-related side effects and adverse reactions (MeSH D064420), Tomography scanners, X-ray computed (MeSH: D015898), Percutaneous coronary interventions (MeSH: D062645), Contrast-induced nephropathy, Post-contrast acute kidney injury
\end{abstract}

\section{Background}

The use of iodinated contrast media $(\mathrm{CM})$ to enhance the imaging visualization of anatomical structures is very frequent. However, in clinical practice, whether the benefits of iodinated CM administration outweigh its potential harms is often unclear [1,2]. Indeed, acute kidney injury (AKI) that follows intravascular administration of $\mathrm{CM}-$ also referred to as contrast-associated AKI (CA-AKI)-is a major concern since it is associated with negative outcomes [3-5]. It occurs in 10-20\% of critically ill patients [6], depending on several clinical factors including the patient's condition but also depending on the definition used [3]. Therefore, clinicians are often puzzled [7]: how

\footnotetext{
${ }^{*}$ Correspondence: lakhal_karim@yahoo.fr

'Service d'Anesthésie-Réanimation, Hôpital Laënnec, Centre Hospitalier Universitaire, Boulevard Jacques-Monod, Saint-Herblain, 44093 Nantes, France

Full list of author information is available at the end of the article
}

to reliably estimate the renal risk of iodinated $\mathrm{CM}$ infusion in a patient? How big is the burden of CM renal toxicity? Are there means to attenuate this renal risk? Should the imaging procedure, even urgent, be cancelled or postponed?

Within the impressive myriad of scientific articles on the subject [8], several points have been recently reappraised. We herein review these significant advances through a typical patient-centred case vignette in the critical care setting. We focused on adults because available data in the paediatric population are very limited.

\section{Main text}

A 55-year-old male with a history of type 2 diabetes mellitus complicated with ischemic heart disease and chronic kidney disease presents to the emergency department with abdominal pain and chills. The first clinical examination also reveals fever, skin original author(s) and the source, provide a link to the Creative Commons licence, and indicate if changes were made. The images or other third party material in this article are included in the article's Creative Commons licence, unless indicated otherwise in a credit line to the material. If material is not included in the article's Creative Commons licence and your intended use is not permitted by statutory regulation or exceeds the permitted use, you will need to obtain permission directly from the copyright holder. To view a copy of this licence, visit http://creativecommons.org/licenses/by/4.0/. The Creative Commons Public Domain Dedication waiver (http://creativeco mmons.org/publicdomain/zero/1.0/) applies to the data made available in this article, unless otherwise stated in a credit line to the data. 
mottling, hypotension and tachycardia. Serum creatinine concentration is $156 \mu \mathrm{mol} . \mathrm{L}^{-1}\left(1.8 \mathrm{mg} \cdot \mathrm{dL} \mathrm{L}^{-1}\right)$. Arterial lactate concentration is 3.7 mmol. $\mathrm{L}^{-1}$. As the attending intensivist, you ask for an abdominal iodinated CM-enhanced computed tomography scanner (CT scan) but the radiologist is worried about the risk of $C A-A K I$.

\section{What is the renal risk of $\mathrm{CM}$ infusion?}

For decades, fear of contrast-induced AKI has been pervasive and, beyond experimental data, this concern was supported by several clinical studies, mainly in the coronary angiography setting [9]. This belief significantly impacts the decision-making process since some patients may be deprived from a potentially beneficial imaging procedure. Fear of contrast-induced AKI has also prompted tremendous costly efforts to find out means to obviate it [10]. Indeed, hundreds of studies assessed all sorts of preventive measures, most of them being ineffective, some being cumbersome, onerous or even harmful [1]. Importantly, the vast majority of studies assessing the renal risk of CM administration did not include a control group, i.e. patients unexposed to $\mathrm{CM}$ but with similar AKI risk factors than exposed patients. Therefore, those uncontrolled studies reported the incidence of AKI following an imaging procedure in critically ill patients rather than the incidence of $\mathrm{CM}$ renal toxicity. In our clinical vignette, at the time of the imaging procedure, the patient cumulates several risk factors of AKI: diabetes, chronic kidney disease, potential sepsis, hypotension, low cardiac output, possible other nephrotoxic medications (Fig. 1) [11]. Which one to blame if AKI develops within the few days after exposure to iodinated CM? Is iodinated CM the culprit? May iodinated $\mathrm{CM}$ contribute to AKI? To what extent? This uncertainty underscores the importance of a control group in order to determine the respective contribution of iodinated $\mathrm{CM}$ and of other risk factors in the development of AKI in the aftermath of the imaging procedure. Hence, among hospitalized patients, meta-analyses of studies including a control group reported that the risk of AKI was similar in patients exposed and unexposed to iodinated CM $[12,13]$. This conclusion was consistent in a subgroup of patients particularly at-risk of AKI (patients with diabetes and chronic kidney disease) [12]. Those findings were confirmed among over 6 million patients of a US nationwide registry [14].

One might question the value of these observational controlled studies with direct comparison of patients having received $\mathrm{CM}$ with those who have not, invoking a selection bias: CM could have been withheld in patients at risk of AKI. Overcoming this issue is not straightforward. Obviously, an interventional study design that

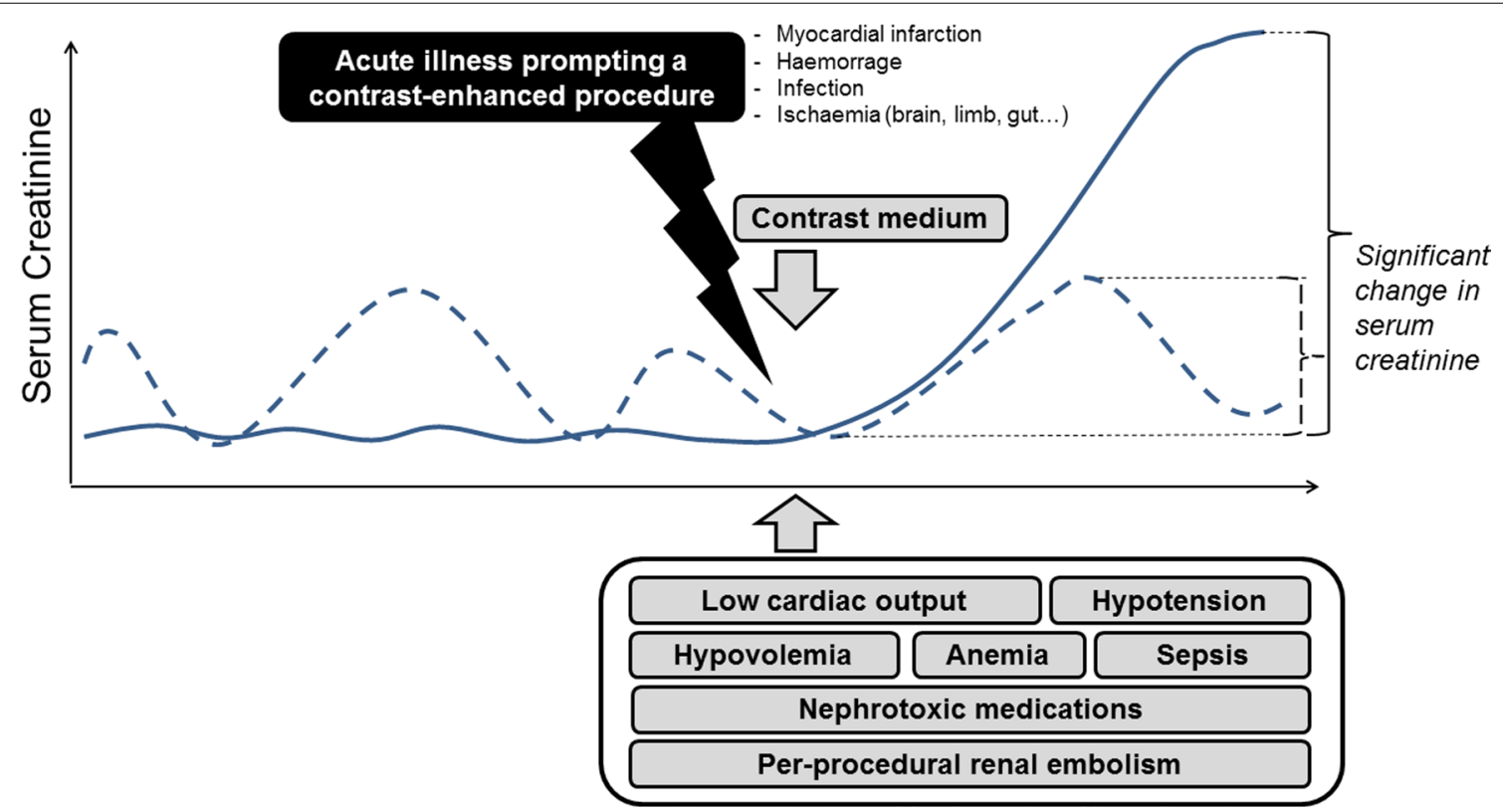

Fig. 1 Typical patterns of significant variations of serum creatinine after contrast media infusion with a special emphasis put on alternative causes of acute kidney injury. The acute illness prompting the contrast-enhanced procedure often comes along with several renal insults. Therefore, incriminating the contrast medium in the subsequent decline in renal function is often purely speculative. In addition, the dotted line displays background significant fluctuations of serum creatinine, even before exposure to contrast media. These examples underscore how the determination of contrast medium-specific contribution in the subsequent impairment in renal function is challenging 
would randomly assign patients to undergo an iodinated CM-enhanced imaging procedure or not would require a very large population [15], may raise ethical concerns and would be source of other biases. Indeed, downgrading the imaging procedure by banning $\mathrm{CM}$ administration may worsen patients' condition and multiple organ failure may arise...including AKI! A comparison of AKI incidence between patients exposed and unexposed to $\mathrm{CM}$ would therefore not discriminate the nephrotoxicity of $\mathrm{CM}$ from the harmfulness of withholding CM. A more advanced observational study design may be a valuable alternative option: matching patients exposed to $\mathrm{CM}$ with unexposed patients having the same baseline risk of receiving $\mathrm{CM}$, using a propensity score approach mimicking randomization to receive $\mathrm{CM}$ or not. Observational studies which adopted this design confirmed that the incidence of AKI after the imaging procedure is similar in propensity score-matched exposed and unexposed patients [12, 14]. Likewise, in the specific setting of the intensive care unit (ICU) where AKI is a major concern since renal risk factors are often combined in the same patient, controlled studies using propensity score-matched analysis also failed to demonstrate that iodinated CM precipitates AKI (Table 1). Indeed, retrospective works including thousands of matched patients did not establish causality between $\mathrm{CM}$ intravenous exposure and AKI, renal replacement therapy or mortality [16-18]. This was confirmed by a meta-analysis among ICU patients using patient-level data [15] and comprising prospective studies $[19,20]$. Similar findings were reported in the emergency department (Table 1).

To assess the specific causal implication of CM among several renal aggressions within hours or days, using a delayed biomarker such as serum creatinine is possibly an imperfect way to proceed. The combination of tissue inhibitor of metalloproteinases-2 (TIMP-2) and insulinlike growth factor binding protein-7 (IGFBP-7) has been proposed as a more specific and sensitive biomarker for early detection of AKI [21]. Interestingly, intravenous $\mathrm{CM}$ infusion did not induce significant changes in urinary [TIMP-2].[IGFBP-7] in ICU patients [22]. This finding may also support the hypothesis of a clinically insignificant toxicity of iodinated CM.

In summary, the renal risk attributable to the intravenous infusion of modern iodinated CM appears, at most, minimal (Table 2). In other words, the widely used term "contrast-induced nephropathy" is often misleading [10]. However, it is unclear whether CM significantly contributes or not to AKI in some very specific populations or during procedures associated with intense kidney exposure to CM (i.e. high systemic dose and/or arterial infusion close to the kidneys). Indeed, patients with preexisting renal impairment at the time of $\mathrm{CM}$ infusion could be more likely to further worsen their renal function if they received iodinated CM $[16,23]$. However, this finding of one study, potentially exposed to selection bias [24], has not been firmly confirmed by other studies [25-28] .

Key message There is increasing evidence that the nephrotoxicity directly attributable to iodinated CM has been exaggerated. One should therefore not refrain from administering CM if deemed necessary.

Pre-contrast CT-scan shows an enlargement of the right kidney with inflammatory changes and a kidney stone in the urinary tract. These findings are consistent with pyelonephritis with pyonephrosis.

Should the intensivist insist on getting a CM-enhanced CT-scan?

Experimental data about the renal toxicity of iodinated CM Several aforementioned controlled studies questioned the clinical relevance of the impact of CM administration on the kidney. However, the in vitro renal toxicity of iodinated CM could hardly be denied, even if many animal models may have poor applicability to humans. CM may be toxic to the kidney through [29]:

- renal ischemia via CM-induced arterial vasoconstriction impairing renal blood flow.

- The release of reactive oxygen species.

- Direct tubular toxicity (osmotic nephrosis, induction of apoptosis, cellular energy failure).

Besides the administered volume, osmolarity of the iodinated $\mathrm{CM}$ is of importance. High-osmolar CM, involved in the first historic CA-AKI descriptions, are more nephrotoxic than modern iso- and low-osmolar $\mathrm{CM}$ and were therefore abandoned [30].

The aforementioned observational controlled studies reporting a similar incidence of AKI in patients exposed and unexposed to CM suggest that modern $\mathrm{CM}$ are of modest clinical impact on renal function. Of note, an alternative interpretation of these studies could be that fear of contrast-induced nephropathy may have prompted a thorough periprocedural management aiming at mitigating the CM-related renal risk: nephroprotective measures, rationale administration of CM (type, volume, timing of administration). Therefore, even if concerns about CM nephrotoxicity will lessen, caution about the renal risk of $\mathrm{CM}$ as well as its non-renal risks (e.g. anaphylaxis, additional radiation exposure) will always remain warranted. 


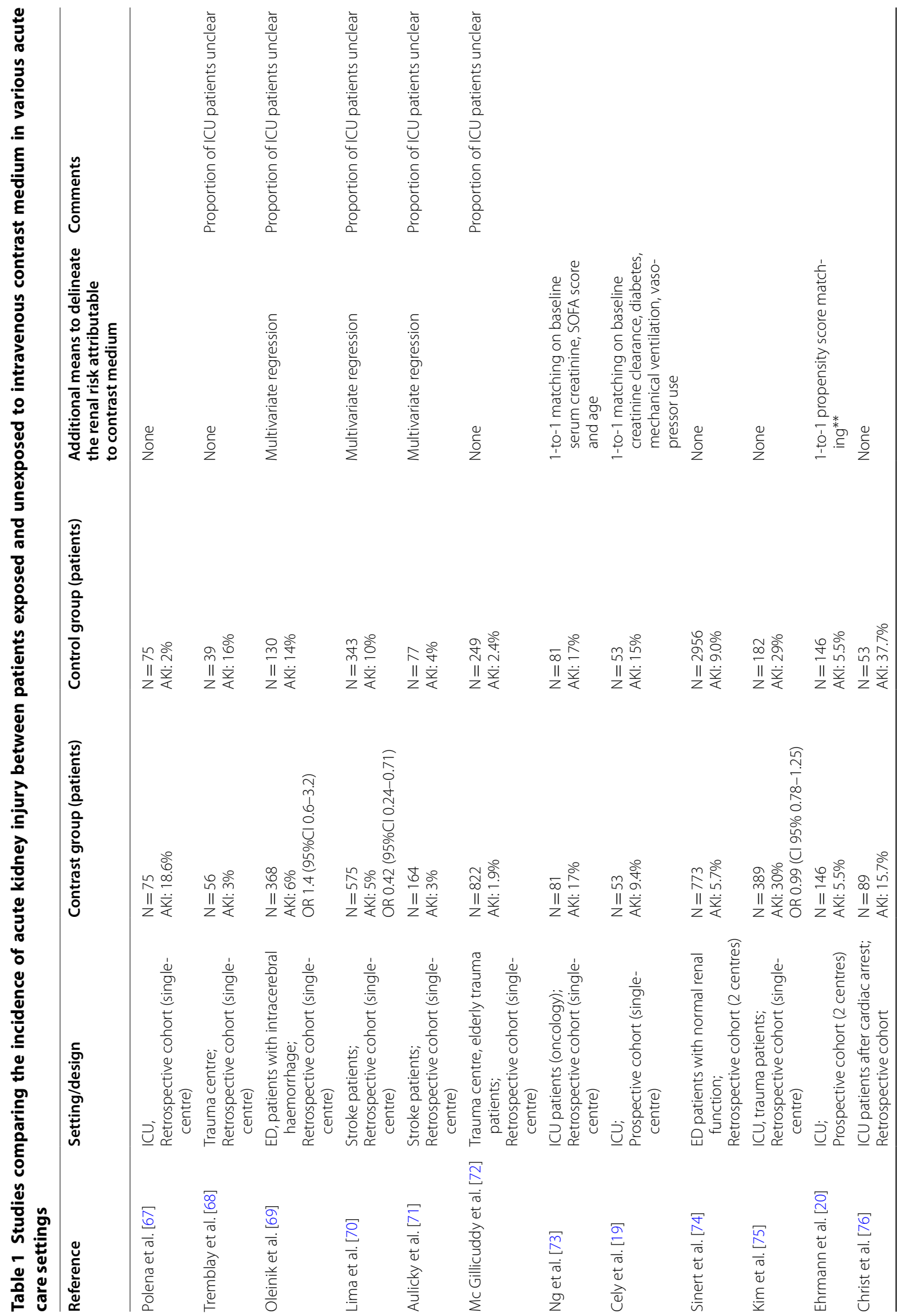




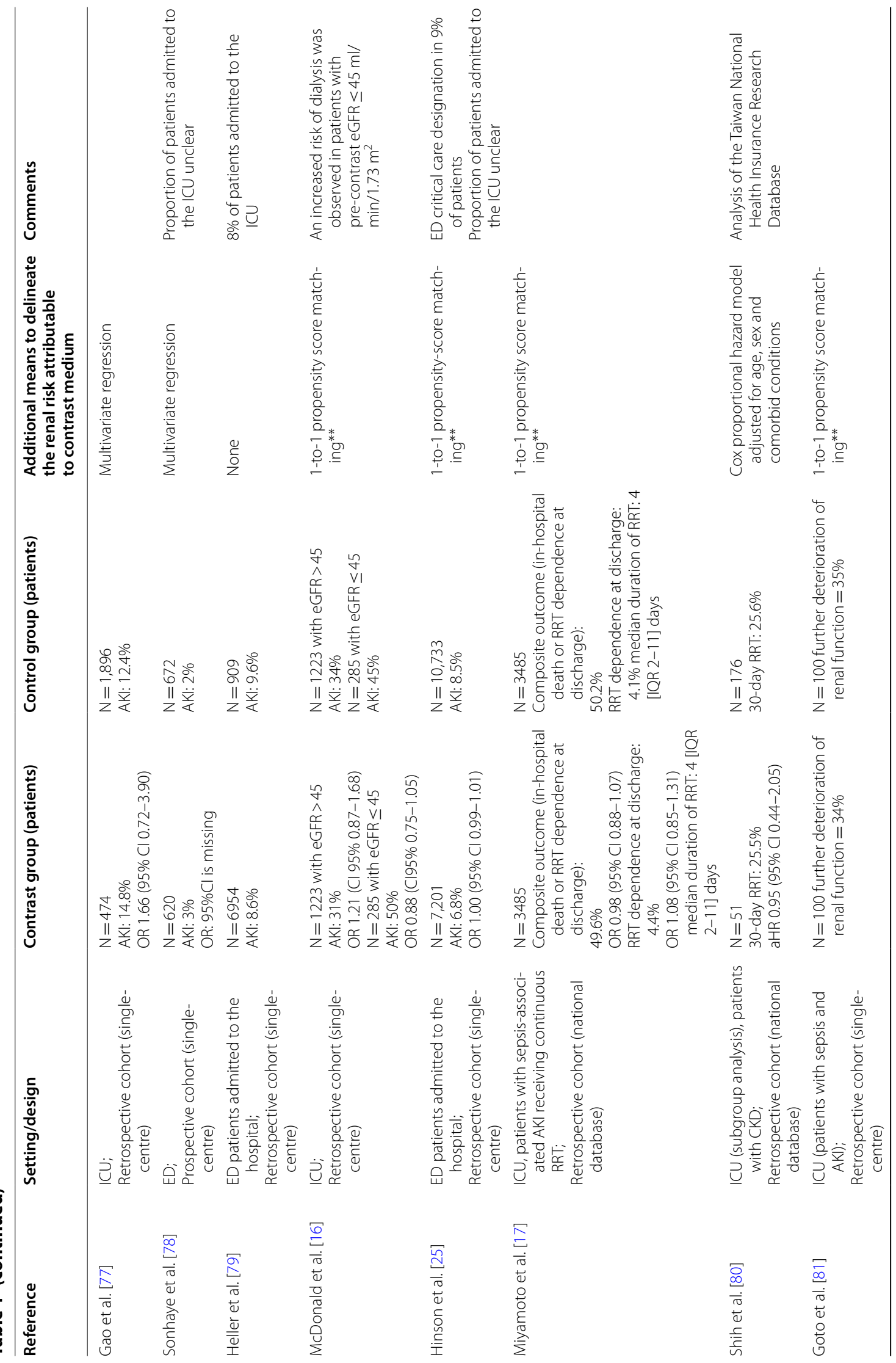




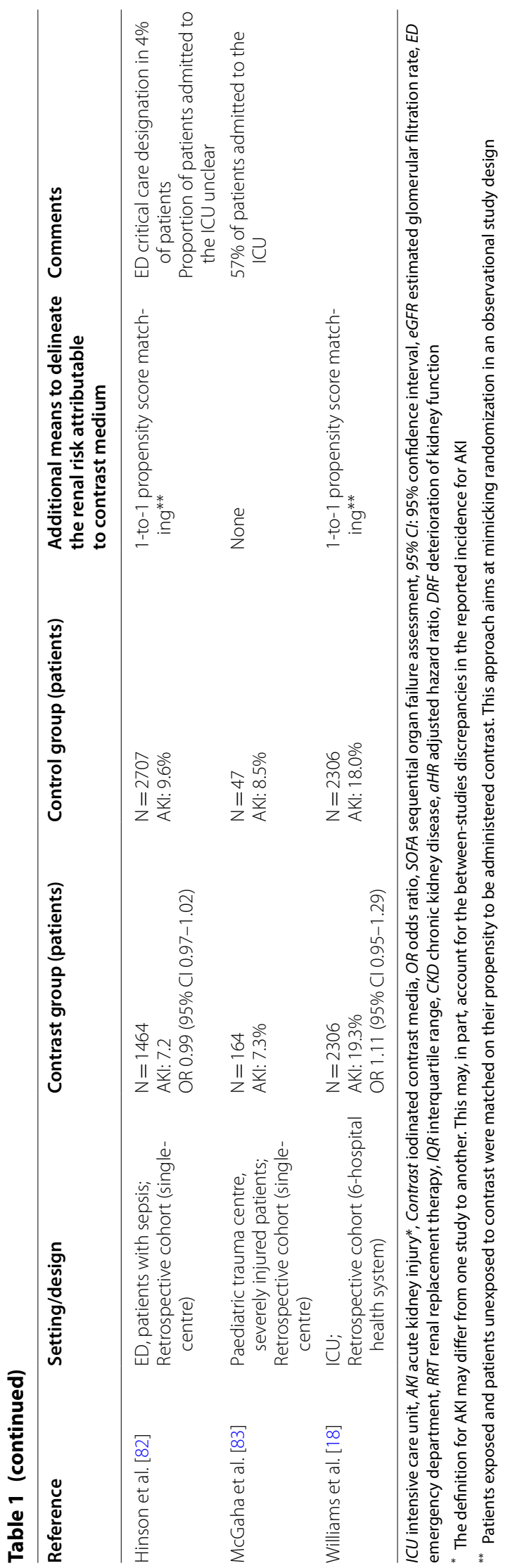




\section{Table 2 Key messages}

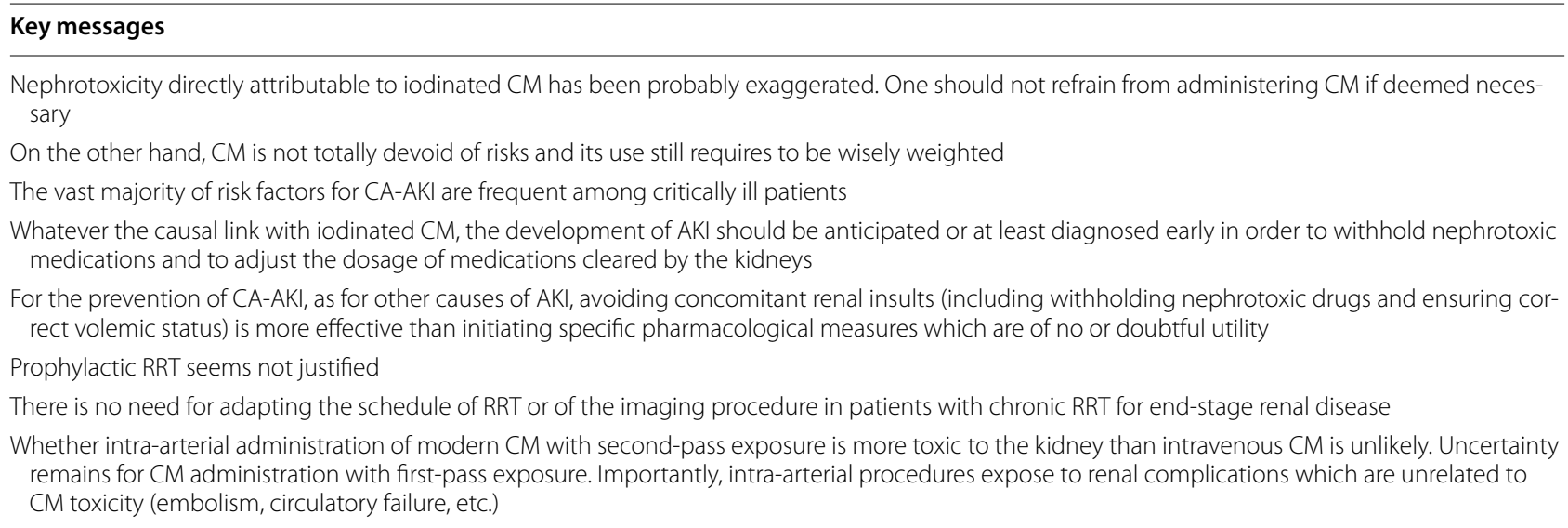

$C M$ contrast medium, $C A-A K I$ contrast-associated acute kidney injury, $A K I$ acute kidney injury, $R R T$ renal replacement therapy

In our clinical vignette of acute pyelonephritis due to urolithiasis, the indication for CM administration could be questioned: unenhanced CT can detect calculi, obstruction, renal enlargement, gas formation and inflammatory masses [31]. It was sufficient for the diagnosis of pyelonephritis with pyonephrosis. Since CMenhanced CT may provide more subtle information [31, 32] but possibly not crucial in the urgent decision-making, the diagnostic yield of CM administration and its therapeutic consequences should be discussed with the radiologist.

Key message Modern CM are less nephrotoxic than older, high-osmolar, iodinated CM. However, considering the renal toxicity of iodinated CM in animal models, besides other related risks (anaphylaxis, additional radiation exposure, etc.), CM cannot be considered totally devoid of risks and should therefore be administered only if necessary.

The radiologist asks you about the patient's risk factors for $C A-A K I$.

\section{Risk factors for CA-AKI}

The use of high-osmolar CM, ionic CM or high-viscosity $\mathrm{CM}$ is nowadays seldom. The infused volume of CM should be limited to as little as possible [33-35]. The renal impact of repeated exposure to $\mathrm{CM}$ within a short period of time should be better evaluated [36].

Patient-related risk factors for CA-AKI are nonspecific, common to other causes of AKI: pre-existing impaired renal function which may be unknown and overlooked, diabetes mellitus, chronic hypertension, advanced age, malignancy, metabolic disorders, anaemia, heart failure, hypovolemia, hypotension, inflammation/sepsis [33-35]. Special emphasis should be put on concurrent nephrotoxic medications [11, 20, 37]. For instance, patients treated with angiotensin-converting enzyme inhibitors or angiotensin receptor blockers are more prone to develop CA-AKI [38]. Anyhow, these drugs are usually withheld when an acute severe illness occurs, irrespective of CM administration, but residual effect may last at the time of the imaging procedure. Periprocedural administration of diuretics has been advocated by some authors as a means to mitigate the renal toxicity of CM (for instance, via the reduction of exposure to $C M$ by increasing urine flow) [39]. However, diuretics promote negative fluid balance and thereby CA-AKI if fluid losses are not promptly replaced [39]. The prescription of some nephrotoxic medications can be hardly avoided as it can be dictated by the acute condition which prompted the imaging procedure such as anti-infective agents, for instance (aminoglycoside, vancomycin, high-dose beta-lactams, amphotericin B, acyclovir, etc.) [11].

The patient is currently treated with metformin. Metformin is the typical example of a drug that may accumulate if a decline in renal function occurs. Dosing adjustment of drugs cleared by the kidney is therefore desirable. To prevent metformin-associated lactic acidosis, although this complication has probably been overestimated [40], metformin should be withheld if a decline in renal function is likely to occur or has occurred [41]. In our clinical vignette, irrespective of CM administration, sepsis-induced AKI was a sufficient reason to withhold metformin.

Unsurprisingly for a critically ill patient, several risk factors for AKI are present in our clinical vignette. Pragmatically, one may consider all critically ill patients at risk of AKI. In addition, common sense dictates that, when a 
patient is admitted to the hospital with high serum creatinine, looking for a pre-existing chronic kidney disease (by enquiring about serum creatinine measurements within the previous weeks/months) is advisable in order to estimate baseline renal function as well as investigating differential diagnosis of possible chronic kidney disease.

Key message The vast majority of the risk factors for CA-AKI are frequent in the critically ill. Whatever the causal link with iodinated CM, the development of AKI should be anticipated or at least diagnosed early in order to withhold non-indispensable nephrotoxic medications and to adjust the dosage of medications cleared by the kidneys.

Whatever, the patient received intravenous CM. A medical student suggests acetylcysteine administration to prevent AKI and ask you about other preventive measures.

\section{CA-AKI pharmacological prophylaxis}

Almost 200 randomized controlled trials (RCTs) tested pharmacological prevention of CA-AKI [42]. More than 42,000 patients were enrolled in these RCTs. N-acetylcysteine $(>6000$ patients), intravenous hydration $(>5000)$, sodium bicarbonate $(\approx 3400)$, statins $(>3000)$ were the most tested among more than 40 tested interventions [42]. For each prevention method, conflicting conclusions were reported, from one study to another and even from one meta-analysis to another [43-45]. Recently, a large RCT $(\approx 5000$ patients at risk of CA-AKI, i.e. with pre-existing impaired renal function) has shown neither a superiority of $\mathrm{N}$-acetylcysteine over placebo nor a superiority of IV sodium bicarbonate $1.26 \%$ over IV saline $0.9 \%$ for a scheduled angiography (coronary or other) [46]. In ICU patients, similar results were reported for $\mathrm{N}$-acetylcysteine [47] and sodium bicarbonate [48] and are therefore not recommended [24]. Of note, in ICU patients with both moderate-to-severe AKI of various causes and severe metabolic acidaemia $(\mathrm{pH} \leq 7.20)$, therapeutic-rather than prophylactic-administration of sodium bicarbonate was associated with a higher incidence of survival by day 28 [49].

Maintaining a correct hydration status is a mainstay of the prevention of AKI [24]. However, it is worth reminding that excessive hydration could be harmful. Indeed, in non-ICU patients ( $>90 \%$ of outpatients) with impaired renal function (estimated glomerular filtration rate of $<60 \mathrm{~mL} / \mathrm{min} / 1.73 \mathrm{~m}^{2}$ ) and undergoing an elective procedure with $\mathrm{CM}$ administration, prophylactic administration of intravenous $0.9 \% \mathrm{NaCl}$ did not reduce the incidence of CA-AKI and $4 \%$ of patients developed signs of congestive heart failure and hydration had to be stopped prematurely, diuretic therapy had to be prompted, and/or hospitalization has been extended [50].

None of the other pharmacological prevention methods was associated with robust evidence of benefit whereas their possible harmfulness should be kept in mind.

Failure to undoubtedly demonstrate positive effects of different prophylactic strategies targeting various pathways of CM renal possible toxicity could be related to the little clinical impact of this toxicity itself with modern CM. It is therefore likely that most studies testing these strategies were underpowered or that the assumptions made about the tested preventive measures were erroneous.

Key message For the prevention of CA-AKI, as for other causes of AKI, avoiding concomitant renal insults (including withholding unnecessary nephrotoxic drugs and ensuring correct volemic status) is more effective than initiating specific pharmacological measures which are of no or doubtful utility.

\section{Before the admission to the ICU, antimicrobial ther- apy was initiated, along with fluid resuscitation and norepinephrine. A ureteral stent has been inserted. The fellow proposes to start renal replacement ther- apy to remove $C M$.}

Renal replacement therapy after contrast administration Besides considering RRT a few days after CM infusion, if severe AKI developed, some authors investigated RRT for the prevention of CA-AKI, i.e. to clear CM from the patient's blood. Hence, prophylactic RRT has been tested in the immediate aftermath of CM infusion or even during the CM-enhanced imaging procedure [51]. Indeed, RRT increases the clearance of iodinated $\mathrm{CM}[51,52]$. Accelerating the withdrawal of CM from the body may be appealing in patients with severe renal dysfunction: the lower the glomerular filtration rate the higher the half-life of iodinated CM, yielding a high concentration of $\mathrm{CM}$ during a protracted period [52]. Iodinated $\mathrm{CM}$ are water-soluble and their extracellular distribution, their limited protein binding and their lack of metabolization could make RRT a suitable means to remove CM from the body [52]. However, despite an effective CM removal, RRT does not reduce the incidence of CA-AKI according to a meta-analysis of 11 single-centres studies among 1010 patients with mild to severe chronic kidney disease (CKD), predominantly undergoing coronary angiography [51]. RRT modality and baseline renal function may matter since prophylactic haemodialysis was even associated 
with a higher risk of CA-AKI in patients with mild CKD as compared with standard medical therapy whereas it was not the case in the scarce studies addressing prophylactic haemofiltration or prophylactic haemodialysis in patients with more severe CKD [51, 53]. Overall, possible explanations for the lack of clear nephroprotective effects of prophylactic RRT are RRT-specific risks at the renal level (blood-membrane contact-related inflammation, systemic hypotension), an insufficient removal of $\mathrm{CM}$ before the onset of renal injury [54] and the lack of clinically significant toxicity of CM.

Key message Prophylactic RRT should not be performed since this overuse of medical resources seems not justified [41].

Another common issue is "when to perform RRT in patients undergoing chronic RRT?". Indeed, scheduling a RRT session immediately after the exposition to CM is a frequent request to nephrologists or intensivists, in an attempt to prevent further renal damage or even extra-renal complications of the delayed excretion of $\mathrm{CM}$. However, there is no evidence of the benefit of this resource mobilizing strategy $[41,55]$.

Key Message Unless the periprocedural management of the imaging procedure was associated with life-threatening fluid overload (not fully related to the CM itself), there is no need for adapting the schedule of RRT or of the imaging procedure in patients with chronic RRT for end-stage renal disease.

Two days later, sepsis is under control, but the patient complains of chest pain. The electrocardiogram reveals an ST segment elevation and serum cardiac troponin has dramatically increased, suggesting acute myocardial infarction (STEMI). A percutaneous coronary intervention (PCI) is considered. Does the renal risk differ between iodinated CM administration for STEMI and for CT scan?

\section{Intra-arterial versus intravenous $\mathrm{CM}$ and renal risk}

Intra-arterial administration of CM is often deemed to be associated with a higher renal toxicity than the intravenous route [35], but this belief is somewhat controversial [56]. When comparing intravenous to intra-arterial CM infusion in the same patient (the patient being his own control), the route of administration does not seem to impact the incidence of post-procedural AKI [57-60], even if these retrospective studies were possibly exposed to bias [33]. Determining the toxicity of $\mathrm{CM}$ with respect to the route of administration cannot be straightforward.
Indeed, beyond this route, the incidence of post-procedural AKI greatly depends on the patient's condition [33]. Indeed, an elective PCI is associated with a markedly lower risk for AKI (1-2\%) than a PCI for STEMI (incidence of 10-20\%) [1]. To complicate matters, AKI following intra-arterial procedures may be related to catheter-related insults to the kidney rather than CM toxicity. For instance, in more than $50 \%$ of PCIs, the placement of guiding catheters is source of scraping debris from the aorta, exposing the kidney to atheromatous emboli-related ischemia [29]. In addition, impaired renal perfusion is not rare in this setting, due to fluid restriction, arrhythmias, myocardial infarction. These events may lead to a decline in renal function that may be erroneously ascribed to the CM itself, not to mention, again, the concurrent conditions and treatments that also affect renal function [61, 62]. Hence, as for the intravenous route, the clinical relevance of $\mathrm{CM}$-induced nephropathy following an intra-arterial administration is controversial. Interestingly, in the specific setting of PCI which is the most studied intra-arterial CM administration, observational controlled studies failed at firmly demonstrating that iodinated CM precipitates AKI. Patients with STEMI exposed to iodinated CM (during PCI) had a similar incidence of AKI than propensity-matched control patients (treated with thrombolysis or without reperfusion) [63]. In patients with non-ST-elevation acute coronary syndrome, one study reported that performing PCI within 2 days of hospital admission was associated with a small increase in the risk of AKI but not in the risk of dialysis or long-term progression to end-stage renal disease [4]. In another study among patients with high renal risk, cardiac angiography was followed by a very low incidence of CA-AKI episodes associated with serious adverse outcomes over a 3-month follow-up [64].

As illustrated by a study unexpectedly reporting a lower rate of $\mathrm{AKI}$ in patients exposed to $\mathrm{CM}$ during PCI than in unexposed patients [14], it is likely that such observational propensity-matched controlled studies tested the renal impact of early invasive management of acute coronary syndrome rather than the renal toxicity of iodinated CM. This illustrates how determining the specific role of CM in CA-AKI is difficult. However, this finding underscores that depriving the patient from an imaging procedure may be more harmful than exposing him to the potential renal toxicity of CM. Again, the renal toxicity of iodinated $\mathrm{CM}-$ or at least of some iodinated $\mathrm{CM}-$ is undeniable, as suggested by some meta-analyses of randomized controlled trials comparing low- and an iso-osmolar CM (iodixanol) in the subgroup of coronary procedures indicating that CA-AKI was more frequent with low-osmolar CM $[65,66]$. This was not the case with intravenous administration [65]. Overall, one may 
consider that, with modern $\mathrm{CM}$, even used intra-arterially, the burden of CA-AKI has been exaggerated [1].

Last, within intra-arterial CM administrations, it is important to distinguish second-pass exposure, in which the $\mathrm{CM}$ reaches the kidneys in a diluted form, from first-pass exposure in which the kidney is exposed to a relatively undiluted $\mathrm{CM}$. The former occurs when $\mathrm{CM}$ is infused in the right heart, the pulmonary arteries, selectively in branches of the suprarenal aorta (carotid, subclavian, brachial, coronary and mesenteric arteries) or in the infrarenal aorta and its branches. The latter occurs when $\mathrm{CM}$ is infused into the left heart, the suprarenal aorta and, selectively, into the renal arteries. In recent guidelines, intra-arterial CM administration with second-pass exposure was considered to have no higher renal toxicity than intravenous CM administration [33]. As aforementioned, there is no strong evidence that, during coronary angiography and PCI which frequently combine both first- and second-pass renal exposure, the toxicity of $\mathrm{CM}$ is higher. However, as a precaution and because of catheterism-related complications, a more thorough prevention of AKI is recommended [33].

Key Message Whether intra-arterial administration of modern CM with second-pass exposure is more toxic to the kidney than intravenous $\mathrm{CM}$ is unlikely. Uncertainty remains for $\mathrm{CM}$ administration with first-pass exposure. Importantly, intra-arterial procedures expose to renal complications which are unrelated to CM toxicity (embolism, circulatory failure, etc.). Intra-arterial procedures can convey important patients benefit per se.

\section{Conclusions}

Despite thousands of papers addressing CA-AKI, several areas of uncertainty persist. However, recent advances tended to shake up some old beliefs about CA-AKI. Indeed, the actual burden of CM toxicity has been exaggerated for years, mostly owing to the coexistence of other renal insults at the time of $\mathrm{CM}$ administration (nephrotoxic medications, sepsis, abnormal circulatory status, renal embolism during intra-arterial procedures, etc.) which predominantly contributed to the decline in renal function in the aftermath of $\mathrm{CM}$ administration. As a consequence of such overrating, beyond flawed epidemiologic data, several studies assessing pharmacological measures to prevent CA-AKI were probably underpowered. Rather than administering drugs of doubtful benefit, a rational use of modern CM and avoidance/limitation of concomitant renal insults should be the mainstays of CA-AKI prevention and treatment.

\section{Abbreviations}

AKI: Acute kidney injury; CA-AKI: Contrast-associated acute kidney injury; CKD: Chronic kidney disease; CM: Contrast medium; CT scan: Computed tomography scanner; ICU: Intensive care unit; PCI: Percutaneous coronary intervention; RCT: Randomized controlled trial; RRT: Renal replacement therapy; [TIMP2].[IGFBP-7]: Combination of tissue inhibitor of metalloproteinases-2 and insulin-like growth factor binding protein-7; STEMI: ST-elevation myocardial infarction.

\section{Acknowledgements}

None

\section{Authors' contributions}

K. Lakhal helped in the conception and in the drafting of this manuscript. V. Robert-Edan helped in the conception and in the drafting of this manuscript. $\mathrm{S}$. Ehrmann helped in the conception and in the drafting of this manuscript. All authors reviewed the final manuscript for important intellectual content. All authors read and approved the final manuscript.

\section{Funding}

None.

Availability of data and material

Not applicable.

Ethics approval and consent to participate

Not applicable.

Consent for publication

Not applicable.

\section{Competing interests}

K. Lakhal has no conflict of interest in connection with the work submitted. In addition, $K L$ received, during the past 3 years, lecture fees from MEDTRONIC (once, in 2017), congress registration fees from SANOFI AVENTIS (once in 2018), travel fees from MSD France (once, in 2017), NOVEX PHARMA (once, in 2016), GILEAD SCIENCES (twice, 2016 and 2017), PFIZER (in 2019 and 2020). $\mathrm{S}$. Ehrmann declares having received consultancies from Aerogen Ltd, La Diffusion Technique Française and Bayer Healthcare, research support from Aerogen Ltd, Fisher \& Paykel Healthcare, Hamilton medical, travel reimbursements from Aerogen Ltd and Fisher \& Paykel. V. Robert-Edan has no conflict of interest in connection with the work submitted.

\section{Author details \\ ${ }^{1}$ Service d'Anesthésie-Réanimation, Hôpital Laënnec, Centre Hospitalier Universitaire, Boulevard Jacques-Monod, Saint-Herblain, 44093 Nantes, France. ${ }^{2}$ Médecine Intensive Réanimation, CIC INSERM 1415, CRICS-TriggerSep Network, CHRU Tours, Tours France and Centre d'étude des Pathologies Res- piratoires INSERM U1100, Université de Tours, Tours, France.}

Received: 7 May 2020 Accepted: 29 October 2020

Published online: 10 November 2020

\section{References}

1. Ehrmann S, Aronson D, Hinson JS. Contrast-associated acute kidney injury is a myth: yes. Intensive Care Med. 2018:44:104-6.

2. Weisbord SD, du Cheryon D. Contrast-associated acute kidney injury is a myth: no. Intensive Care Med. 2018;44:107-9.

3. Lakhal K, Ehrmann S, Chaari A, Laissy J-P, Régnier B, Wolff M, et al. Acute kidney injury network definition of contrast-induced nephropathy in the critically ill: incidence and outcome. J Crit Care. 2011;26:593-9.

4. James MT, Samuel SM, Manning MA, Tonelli M, Ghali WA, Faris P, et al. Contrast-induced acute kidney injury and risk of adverse clinical outcomes after coronary angiography: a systematic review and metaanalysis. Circ Cardiovasc Interv. 2013;6:37-43.

5. Mitchell AM, Kline JA, Jones AE, Tumlin JA. Major adverse events one year after acute kidney injury after contrast-enhanced computed tomography. Ann Emerg Med. 2015;66(267-274):e4. 
6. Ehrmann S, Pajot O, Lakhal K. Néphropathie induite par les produits de contraste iodés en réanimation. Réanimation. 2012;21:463-73.

7. Lakhal K, Ehrmann S, Robert-Edan V. lodinated contrast medium renal toxicity: the phantom menace or much ado about nothing? Crit Care Med. 2017:45:e745-6.

8. Lameire N, Kellum JA, KDIGO AKI Guideline Work Group. Contrastinduced acute kidney injury and renal support for acute kidney injury: a KDIGO summary (Part 2). Crit Care. 2013;17:205.

9. McCullough PA. Acute kidney injury with iodinated contrast. Crit Care Med. 2008;36:5204-211.

10. Lakhal K, Robert-Edan V, Ehrmann S. In the name of contrast-induced acute kidney injury.... Chest. 2020;157:751-2.

11. Ehrmann S, Helms J, Joret A, Martin-Lefevre L, Quenot J-P, Herbrecht $J-E$, et al. Nephrotoxic drug burden among 1001 critically ill patients: impact on acute kidney injury. Ann Intensive Care. 2019;9:106.

12. McDonald JS, McDonald RJ, Comin J, Williamson EE, Katzberg RW Murad $\mathrm{MH}$, et al. Frequency of acute kidney injury following intravenous contrast medium administration: a systematic review and metaanalysis. Radiology. 2013;267:119-28.

13. Aycock RD, Westafer LM, Boxen JL, Majlesi N, Schoenfeld EM, Bannuru RR. Acute kidney injury after computed tomography: a meta-analysis. Ann Emerg Med. 2018;71(44-53):e4.

14. Wilhelm-Leen E, Montez-Rath ME, Chertow G. Estimating the risk of radiocontrast-associated nephropathy. J Am Soc Nephrol. 2017;28:653-9.

15. Ehrmann S, Quartin A, Hobbs BP, Robert-Edan V, Cely C, Bell C, et al. Contrast-associated acute kidney injury in the critically ill: systematic review and Bayesian meta-analysis. Intensive Care Med. 2017:43:785-94.

16. McDonald JS, McDonald RJ, Williamson EE, Kallmes DF, Kashani K. Postcontrast acute kidney injury in intensive care unit patients: a propensity score-adjusted study. Intensive Care Med. 2017:43:774-84.

17. Miyamoto Y, Iwagami $M$, Aso $S$, Yasunaga $H$, Matsui $H$, Fushimi $K$, et al. Association between intravenous contrast media exposure and nonrecovery from dialysis-requiring septic acute kidney injury: a nationwide observational study. Intensive Care Med. 2019;45:1570-9.

18. Williams L-MS, Walker GR, Loewenherz JW, Gidel LT. Association of contrast and acute kidney injury in the critically ill: a propensity-matched study. Chest. 2020;157:866-76.

19. Cely CM, Schein RMH, Quartin AA. Risk of contrast induced nephropathy in the critically ill: a prospective, case matched study. Crit Care. 2012;16:R67.

20. Ehrmann S, Badin J, Savath L, Pajot O, Garot D, Pham T, et al. Acute kidney injury in the critically ill: is iodinated contrast medium really harmful? Crit Care Med. 2013;41:1017-26.

21. Kashani K, Al-Khafaji A, Ardiles T, Artigas A, Bagshaw SM, Bell M, et al. Discovery and validation of cell cycle arrest biomarkers in human acute kidney injury. Crit Care. 2013;17:R25.

22. Rouve E, Lakhal K, Salmon Gandonnière C, Jouan Y, Bodet-Contentin L, Ehrmann S. Lack of impact of iodinated contrast media on kidney cellcycle arrest biomarkers in critically ill patients. BMC Nephrol. 2018;19:308.

23. Davenport MS, Khalatbari S, Cohan RH, Dillman JR, Myles JD, Ellis JH. Contrast material-induced nephrotoxicity and intravenous low-osmolality iodinated contrast material: risk stratification by using estimated glomerular filtration rate. Radiology. 2013;268:719-28.

24. Joannidis M, DrumI W, Forni LG, Groeneveld ABJ, Honore PM, Hoste E, et al. Prevention of acute kidney injury and protection of renal function in the intensive care unit: update 2017: expert opinion of the Working Group on Prevention, AKI section, European Society of Intensive Care Medicine. Intensive Care Med. 2017:43:730-49.

25. Hinson JS, Ehmann MR, Fine DM, Fishman EK, Toerper MF, Rothman RE, et al. Risk of acute kidney injury after intravenous contrast media administration. Ann Emerg Med. 2017;69(577-586):e4.

26. McDonald JS, McDonald RJ, Carter RE, Katzberg RW, Kallmes DF, Williamson EE. Risk of intravenous contrast material-mediated acute kidney injury: a propensity score-matched study stratified by baseline-estimated glomerular filtration rate. Radiology. 2014;271:65-73.

27. McDonald JS, McDonald RJ, Lieske JC, Carter RE, Katzberg RW, Williamson $\mathrm{EE}$, et al. Risk of acute kidney injury, dialysis, and mortality in patients with chronic kidney disease after intravenous contrast material exposure. Mayo Clin Proc. 2015;90:1046-53.
28. Petek BJ, Bravo PE, Kim F, de Boer IH, Kudenchuk PJ, Shuman WP, et al. Incidence and risk factors for postcontrast acute kidney injury in survivors of sudden cardiac arrest. Ann Emerg Med. 2016;67(469-476):e1.

29. Tumlin J, Stacul F, Adam A, Becker CR, Davidson C, Lameire N, et al. Pathophysiology of contrast-induced nephropathy. Am J Cardiol. 2006;98:14K-20K.

30. Barrett BJ, Carlisle EJ. Metaanalysis of the relative nephrotoxicity of highand low-osmolality iodinated contrast media. Radiology. 1993;188:171-8.

31. Stunell H, Buckley O, Feeney J, Geoghegan T, Browne RFJ, Torreggiani WC Imaging of acute pyelonephritis in the adult. Eur Radiol. 2007;17:1820-8.

32. Nikolaidis P, Dogra VS, Goldfarb S, Gore JL, Harvin HJ, et al. ACR Appropriateness Criteria ${ }^{\circledR}$ acute pyelonephritis. J Am Coll Radiol. 2018;15:S232-9.

33. van der Molen AJ, Reimer P, Dekkers IA, Bongartz G, Bellin M-F, Bertolotto $\mathrm{M}$, et al. Post-contrast acute kidney injury - Part 1: definition, clinical features, incidence, role of contrast medium and risk factors: recommendations for updated ESUR Contrast Medium Safety Committee guidelines. Eur Radiol. 2018a;28:2845-55.

34. Pannu N, Wiebe N, Tonelli M. Alberta kidney disease network. Prophylaxis strategies for contrast-induced nephropathy. JAMA. 2006;295:2765-79.

35. Acute Kidney Injury (AKI)—KDIGO. https://Kdigo.org/quidelines/acute -kidney-injury/

36. Winther $\mathrm{S}$, Svensson $M$, Jørgensen $H S$, Birn $H$, Bøtker $H E$, Ivarsen $P$, et al. Repeated contrast administration is associated with low risk of postcontrast acute kidney injury and long-term complications in patients with severe chronic kidney disease. Am J Transplant. 2016;16:897-907.

37. Ho Y-F, Hsieh K-L, Kung F-L, Wu F-LL, Hsieh L-L, Chou H, et al. Nephrotoxic polypharmacy and risk of contrast medium-induced nephropathy in hospitalized patients undergoing contrast-enhanced CT. AJR Am J Roentgenol. 2015;205:703-8.

38. Rim MY, Ro H, Kang WC, Kim AJ, Park H, Chang JH, et al. The effect of renin-angiotensin-aldosterone system blockade on contrast-induced acute kidney injury: a propensity-matched study. Am J Kidney Dis. 2012;60:576-82.

39. Fähling M, Seeliger E, Patzak A, Persson PB. Understanding and preventing contrast-induced acute kidney injury. Nat Rev Nephrol. 2017;13:169-80.

40. Inzucchi SE, Lipska KJ, Mayo H, Bailey CJ, McGuire DK. Metformin in patients with type 2 diabetes and kidney disease: a systematic review. JAMA. 2014;312:2668-75.

41. van der Molen AJ, Reimer P, Dekkers IA, Bongartz G, Bellin M-F, Bertolotto $M$, et al. Post-contrast acute kidney injury. Part 2: risk stratification, role of hydration and other prophylactic measures, patients taking metformin and chronic dialysis patients: recommendations for updated ESUR Contrast Medium Safety Committee guidelines. Eur Radiol. 2018b;28:2856-69.

42. Ahmed K, McVeigh T, Cerneviciute R, Mohamed S, Tubassam M, Karim M, et al. Effectiveness of contrast-associated acute kidney injury prevention methods; a systematic review and network meta-analysis. BMC Nephrol. 2018;19:323.

43. Xu R, Tao A, Bai Y, Deng Y, Chen G. Effectiveness of N-acetylcysteine for the prevention of contrast-induced nephropathy: a systematic review and meta-analysis of randomized controlled trials. J Am Heart Assoc. 2016;5:e003968.

44. Li J-X, Jin E-Z, Yu L-H, Li Y, Liu N-N, Dong Y-M, et al. Oral N-acetylcysteine for prophylaxis of contrast-induced nephropathy in patients following coronary angioplasty: a meta-analysis. Exp Ther Med. 2017;14:1568-76.

45. Loomba RS, Shah PH, Aggarwal S, Arora RR. Role of N-acetylcysteine to prevent contrast-induced nephropathy: a meta-analysis. Am J Ther. 2016:23:e172-183.

46. Weisbord SD, Gallagher M, Jneid H, Garcia S, Cass A, Thwin S-S, et al. Outcomes after angiography with sodium bicarbonate and acetylcysteine. N Engl J Med. 2018;378:603-14.

47. Palli E, Makris D, Papanikolaou J, Garoufalis G, Tsilioni I, Zygoulis P, et al. The impact of $\mathrm{N}$-acetylcysteine and ascorbic acid in contrast-induced nephropathy in critical care patients: an open-label randomized controlled study. Crit Care. 2017;21:269.

48. Valette X, Desmeulles I, Savary B, Masson R, Seguin A, Sauneuf B, et al. Sodium bicarbonate versus sodium chloride for preventing contrastassociated acute kidney injury in critically ill patients: a randomized controlled trial. Crit Care Med. 2017:45:637-44.

49. Jaber S, Paugam C, Futier E, Lefrant J-Y, Lasocki S, Lescot T, et al. Sodium bicarbonate therapy for patients with severe metabolic acidaemia in the 
intensive care unit (BICAR-ICU): a multicentre, open-label, randomised controlled, phase 3 trial. Lancet. 2018;392:31-40.

50. Nijssen EC, Rennenberg RJ, Nelemans PJ, Essers BA, Janssen MM, Vermeeren MA, et al. Prophylactic hydration to protect renal function from intravascular iodinated contrast material in patients at high risk of contrast-induced nephropathy (AMACING): a prospective, randomised, phase 3, controlled, open-label, non-inferiority trial. Lancet. 2017;389:1312-22.

51. Cruz DN, Goh CY, Marenzi G, Corradi V, Ronco C, Perazella MA. Renal replacement therapies for prevention of radiocontrast-induced nephropathy: a systematic review. Am J Med. 2012;125(66-78):e3.

52. Morcos SK, Thomsen HS, Webb JAW. Contrast media safety committee of the European society of urogenital radiology (ESUR). Dialysis and contrast media. Eur Radiol. 2002;12:3026-30.

53. Choi M-J, Yoon J-W, Han S-J, Choi H-H, Song Y-R, Kim S-G, et al. The prevention of contrast-induced nephropathy by simultaneous hemofiltration during coronary angiographic procedures: a comparison with periprocedural hemofiltration. Int J Cardiol. 2014;176:941-5.

54. Gabutti L, Marone C, Monti M, Malfanti M, Zwahlen U, Pasotti E, et al. Does continuous venovenous hemodiafiltration concomitant with radiological procedures provide a significant and safe removal of the iodinated contrast ioversol? Blood Purif. 2003;21:152-7.

55. Deray G. Dialysis and iodinated contrast media. Kidney Int Suppl. 2006;69:S25-29.

56. Nyman U, Almén T, Jacobsson B, Aspelin P. Are intravenous injections of contrast media really less nephrotoxic than intra-arterial injections? Eur Radiol. 2012;22:1366-71.

57. Kooiman J, Le Haen PA, Gezgin G, de Vries J-PP, Boersma D, Brulez HF, et al. Contrast-induced acute kidney injury and clinical outcomes after intra-arterial and intravenous contrast administration: risk comparison adjusted for patient characteristics by design. Am Heart J. 2013;165:793799.e1.

58. Tong GE, Kumar S, Chong KC, Shah N, Wong MJ, Zimmet JM, et al. Risk of contrast-induced nephropathy for patients receiving intravenous vs. intra-arterial iodixanol administration. Abdom Radiol. 2016;41:91-9.

59. McDonald JS, Leake CB, McDonald RJ, Gulati R, Katzberg RW, Williamson EE, et al. Acute kidney injury after intravenous versus intra-arterial contrast material administration in a paired cohort. Investig Radiol. 2016:51:804-9.

60. Karlsberg RP, Dohad SY, Sheng R, lodixanol Peripheral Computed Tomographic Angiography Study Investigator Panel. Contrast mediuminduced acute kidney injury: comparison of intravenous and intraarterial administration of iodinated contrast medium. J Vasc Interv Radiol. 2011;22:1159-65.

61. Newhouse JH, Kho D, Rao QA, Starren J. Frequency of serum creatinine changes in the absence of iodinated contrast material: implications for studies of contrast nephrotoxicity. AJR Am J Roentgenol. 2008;191:376-82.

62. Bruce RJ, Djamali A, Shinki K, Michel SJ, Fine JP, Pozniak MA. Background fluctuation of kidney function versus contrast-induced nephrotoxicity. AJR Am J Roentgenol. 2009;192:711-8.

63. Caspi O, Habib M, Cohen Y, Kerner A, Roguin A, Abergel E, et al. Acute kidney injury after primary angioplasty: is contrast-induced nephropathy the culprit? J Am Heart Assoc. 2017:6:e005715.

64. Weisbord SD, Palevsky PM, Kaufman JS, Wu H, Androsenko M, Ferguson $\mathrm{RE}$, et al. Contrast-associated acute kidney injury and serious adverse outcomes following angiography. J Am Coll Cardiol. 2020;75:1311-20.

65. Dong M, Jiao Z, Liu T, Guo F, Li G. Effect of administration route on the renal safety of contrast agents: a meta-analysis of randomized controlled trials. J Nephrol. 2012;25:290-301.

66. McCullough PA, Brown JR. Effects of intra-arterial and intravenous isoosmolar contrast medium (iodixanol) on the risk of contrast-induced acute kidney injury: a meta-analysis. Cardiorenal Med. 2011;1:220-34.

67. Polena S, Yang S, Alam R, Gricius J, Gupta JR, Badalova N, et al. Nephropathy in critically III patients without preexisting renal disease. Proc West Pharmacol Soc. 2005;48:134-5.
68. Tremblay LN, Tien H, Hamilton P, Brenneman FD, Rizoli SB, Sharkey PW, et al. Risk and benefit of intravenous contrast in trauma patients with an elevated serum creatinine. J Trauma. 2005;59:1162-6 (discussion 1166-1167).

69. Oleinik A, Romero JM, Schwab K, Lev MH, Jhawar N, Delgado Almandoz $J E$, et al. CT angiography for intracerebral hemorrhage does not increase risk of acute nephropathy. Stroke. 2009:40:2393-7.

70. Lima FO, Lev MH, Levy RA, Silva GS, Ebril M, de Camargo EC, et al. Functional contrast-enhanced CT for evaluation of acute ischemic stroke does not increase the risk of contrast-induced nephropathy. AJNR Am J Neuroradiol. 2010;31:817-21.

71. Aulicky P, Mikulík R, Goldemund D, Reif M, Dufek M, Kubelka T. Safety of performing $C T$ angiography in stroke patients treated with intravenous thrombolysis. J Neurol Neurosurg Psychiatry. 2010;81:783-7.

72. McGillicuddy EA, Schuster KM, Kaplan LJ, Maung AA, Lui FY, Maerz LL, et al. Contrast-induced nephropathy in elderly trauma patients. J Trauma. 2010;68:294-7.

73. Ng CS, Shaw AD, Bell CS, Samuels JA. Effect of IV contrast medium on renal function in oncologic patients undergoing CT in ICU. AJR Am J Roentgenol. 2010;195:414-22.

74. Sinert R, Brandler E, Subramanian RA, Miller AC. Does the current definition of contrast-induced acute kidney injury reflect a true clinical entity? Acad Emerg Med. 2012;19:1261-7.

75. Kim DY, Kobayashi L, Costantini TW, Chang D, Fortlage D, Curry T, et al. Is contrast exposure safe among the highest risk trauma patients? J Trauma Acute Care Surg. 2012;72:61-6 (discussion 66-67).

76. Christ M, Auenmüller Kl, Amirie S, Brand M, Sasko BM, Trappe HJ. Acute kidney injury and renal replacement therapy in victims from out-ofhospital cardiac arrest with administration of contrast agent. Herzschrittmacherther Elektrophysiol. 2016;27:6-14.

77. Gao J, Zhang M, Fang G, Ye L, Tang W. Risk and harm of contrast induced nephropathy in critically ill patients. Zhonghua Wei Zhong Bing Ji Jiu Yi Xue. 2015;27:366-70.

78. Sonhaye L, Kolou B, Tchaou M, Amadou A, Assih K, N'Timon B, et al. Intravenous contrast medium administration for computed tomography scan in emergency: a possible cause of contrast-induced nephropathy. Radiol Res Pract. 2015;2015:805786.

79. Heller M, Krieger P, Finefrock D, Nguyen T, Akhtar S. Contrast CT scans in the emergency department do not increase risk of adverse renal outcomes. West J Emerg Med. 2016;17:404-8.

80. Shih Y-J, Kuo Y-T, Ho C-H, Wu C-C, Ko C-C. Incidence and risk of dialysis therapy within 30 days after contrast enhanced computed tomography in patients coded with chronic kidney disease: a nation-wide, populationbased study. PeerJ. 2019;7:e7757.

81. Goto Y, Koyama K, Katayama S, Tonai K, Shima J, Koinuma T, et al. Influence of contrast media on renal function and outcomes in patients with sepsis-associated acute kidney injury: a propensity-matched cohort study. Crit Care. 2019;23:249.

82. Hinson JS, Al Jalbout N, Ehmann MR, Klein EY. Acute kidney injury following contrast media administration in the septic patient: a retrospective propensity-matched analysis. J Crit Care. 2019;51:111-6.

83. McGaha PK, Johnson J, Garwe T, Sarwar Z, Motghare P, Daly W, et al. Computed tomography with intravenous contrast is not associated with development of acute kidney injury in severely injured pediatric patients. Am Surg. 2019;85:e1-5.

\section{Publisher's Note}

Springer Nature remains neutral with regard to jurisdictional claims in published maps and institutional affiliations. 